\title{
Turbulence combustion closure model based on the Eddy dissipation concept for large eddy simulation
}

\author{
B. Panjwani ${ }^{1}$, I. S. Ertesvåg ${ }^{1}$, A. Gruber ${ }^{2}$ \& K. E. Rian ${ }^{3}$ \\ ${ }^{1}$ Department of Energy and Process Engineering, \\ Norwegian University of Science and Technology(NTNU), Norway \\ ${ }^{2}$ SINTEF Energy Research, Trondheim, Norway \\ ${ }^{3}$ Computational Industry Technologies (ComputIT), Norway
}

\begin{abstract}
Modeling of turbulence-chemistry interaction is still a challenge. Turbulence modeling with Large Eddy Simulation (LES) has been matured enough for industrial problems. In LES eddies up to the filter width are resolved on the grid scales, but the fine structures where combustion takes place are still not resolved, which calls for combustion modeling in LES. Combustion closure in LES is achieved through a Turbulence Chemistry Interaction Model (TCIM). Most of the developed TCIM are based on the already existing RANS model. In the present study, a TCIM based on the Eddy Dissipation Concept (EDC) is proposed for large eddy simulation. The model is formulated from subgrid viscosity and filtered strain rate tensor. EDC model constants are modified to account for the partial energy cascading in LES. The other model used in this study is the steady state Flamelet model. Another issue with reacting flows is the solution of the pressure correction Poisson's equation with density time derivative term, which causes severe time constraint per iteration. Density time derivative is the most destabilizing part of the calculation when the density from equation of state is used. In the present study density is formulated from species mass fraction, which is numerically stable and computationally less expensive. LES of the H2/N2 "FlameH3" non-premixed unconfined turbulent jet flame is performed using LESEDC and Flamelet model. The Reynolds number based on nozzle diameter and jet bulk velocity is 10,000 . The chemistry used for LES-EDC model is a fastchemistry. Results of the simulations in the form of means and variances of
\end{abstract}


velocity and scalars are compared to experimental data. All these quantities are in satisfactory agreement with experiments.

Keywords: LES, EDC, Flame H3, FLOWSI.

\section{Introduction}

Turbulence Chemistry Interaction Model (TCIM) is an approach where a combustion model for reaction rates is developed considering the major aspects of turbulence. In laminar flows a direct closure of the reaction rate is achieved by the Arrhenius reaction rate equation, but in turbulent flows having fluctuations, the direct closure leads to a difficulty due to non-linearity of the Arrhenius term. The challenging problem in the turbulent combustion is the wide range of length and time scales. The direct closure of a source term is only possible through Direct numerical simulation (DNS). DNS resolves all the reactive length scales, which makes DNS computationally expensive. Another approach is Large Eddy Simulation (LES), where large geometrically-dependent energy carrying eddies are resolved on the grid scales(GS), whereas effects of the smaller, more universal isotropic scales are modeled using a sub-grid scale (SGS) models. LES is a promising tool for understanding the physics of unsteady turbulent flows at comparatively reduced cost. Performance of the TCIM depends on the turbulence modeling. An accurate prediction of the turbulence leads to the better prediction of the combustion characteristics. In LES the small dissipative eddies where combustion takes place are not resolved on the grid scale. This calls for a combustion model in LES also, the combustion models in LES do not differ significantly from the already existing RANS models. An advantage with LES is that it captures the instantaneous quantities and mixing more accurately than RANS do.

Most widely used approaches for turbulence chemistry interaction are, a conserved variable approach and Eddy Dissipation Concept (EDC). In the conserved variable approach a transport equation for the conserved variable such as mixture fraction is solved. The mixture fraction represents the mixed ness of the fuel and oxidizer. In this approach turbulence and local equivalence ratio are represented by filtered mixture fraction, mixture fraction variance and scalar dissipation rate. Chemical kinetic is coupled with a flamelet equation which is solved in the mixture fraction space. A turbulence-chemistry interaction model based on presumed probability density functions (PDF) was presented by Landenfeld [1], which was capable of capturing major and minor species distribution features in turbulent diffusion flames. Branley and Jones [2], Forkel and Janicka [3] carried out a LES of hydrogen diffusion flame using the equilibrium chemistry model. Kempf [4] and Kempf et al. [5, 6] performed LES studies of the Sandia Flame D, a Bluff Body flame, a hydrogen diffusion flame and a counter flow diffusion flame using the steady state flamelet model and they obtained satisfactory agreement with experiments. A LES of the piloted Sandia-D flame using an unsteady Lagrangian flamelet model was performed by the Pitsch and Steiner [7] and they achieved an excellent agreement even 
for minor species. The unsteady flamelet modeling is an expensive approach compared to the steady state approach, but some of the important unsteady phenomena such as extinction, re-ignition is possible through the unsteady approach. McMurtry et al. [8] formulated the Liner Eddy Model (LEM) developed by Kerstein [9] for LES reacting flows.

$\mathrm{Hu}$ et al. [10] carried out a LES simulation of swirling methane/air diffusion flames using the Second Order Moment (SOM) and EDC models. In SOM model the Arrhenius reaction rate equation was used for the direct closure of the source term. The non-linear terms, which arise due to the closure of the Arrhenius reaction rate equations, were modeled. The correlation functions between the temperaturespecies mass fraction and the fuel-oxidizer mass fraction were approximated using the gradient approach. The reaction mechanism used for the simulation was a single step. The SOM model also introduced some problem dependent constants. The predictions with SOM model were satisfactory but the predictions with EDC model did not agree well with the experimental results. The reason for the unsatisfactory prediction was that the EDC model employed by $\mathrm{Hu}$ et al. [10] was the earlier version of EDC model proposed by Magnussen and Hjertager [11], which uses the model constants based on the RANS. The model does not account for the fine structure region and mass fraction also. Yaga et al. [12] carried out a LES study of methane/air diffusion flame using a combustion model which was a combination of the Arrhenius reaction rate and EDC model. The overall reaction rate was the minimum of the Arrhenius reaction rate and the EDC model. The obtained results compared fairly well with experiments except for the CO concentration, that is because of the simple CO mechanism was used in their study. In the present study, a turbulence-chemistry interaction model based on EDC is extended from RANS to LES. A Proposal for modifying the model constant based on the partial cascading is discussed. Present methodology is validated by performing a Large Eddy simulation of the hydrogen diffusion flame "FlameH3" at a Reynolds number of 10,000. A formulation for density based on conservation of species mass fraction is also proposed [13].

\subsection{Governing filtered equations}

The governing filtered equation for LES are

The continuity equation

$$
\frac{\partial \bar{\rho}}{\partial t}+\frac{\partial \bar{\rho} \widetilde{u}_{i}}{\partial x_{i}}=0
$$

The momentum equation

$$
\frac{\partial \bar{\rho} \widetilde{u}_{i}}{\partial t}+\frac{\partial \bar{\rho} \widetilde{u}_{i} \widetilde{u}_{j}}{\partial x_{j}}=-\frac{\partial \bar{p}}{\partial x_{j}}+\frac{\partial}{\partial x_{j}}\left(\mu\left(\frac{\partial \widetilde{u}_{i}}{\partial x_{j}}+\frac{\partial \widetilde{u}_{j}}{\partial x_{i}}\right)\right)-\frac{\partial \tau_{s g s}}{\partial x_{j}}
$$

The mass fraction equation for species $i$

$$
\frac{\partial \bar{\rho} \widetilde{Y}_{i}}{\partial t}+\frac{\partial \bar{\rho} \widetilde{Y}_{i} \widetilde{u}_{j}}{\partial x_{j}}=\frac{\partial}{\partial x_{j}}\left(\bar{\rho} D_{i}\left(\frac{\partial \widetilde{Y}_{i}}{\partial x_{j}}\right)\right)+\bar{\rho} \widetilde{\omega}_{i}
$$


where $\bar{\rho}$ is a (Favre) filtered density, $\widetilde{u_{i}}$ is a filtered velocity component, $\bar{p}$ is pressure $\widetilde{Y}_{i}$ is a species mass fraction for species i, $\mu$ is the dynamic viscosity, $t$ is time, $\tau_{s g s}$ is subgrid stress and $\widetilde{\omega}_{i}$ is the chemical source term. The incompressible governing equations Eqs (1-3) were discretized using the finite volume method (FVM) on a staggered cylindrical grid. A pressure correction Poisson's equation was solved at every time steps, and intermediate velocities and pressure were corrected. Now pressure corrected velocity field is divergence free. The Total Variation Diminishing (TVD) scheme was used for discretizing the convective terms in the species transport equation, and the second order central differencing scheme was used for discretizing the convective terms in the momentum equations. The diffusive terms were discretized using the second order central differencing scheme. The TVD schemes are preferred for discretizing the conserved variables because of their bounded nature. These schemes do not produce undershoot and overshoot in the conserved variables. A scheme is TVD if the total variations in the conserved variable is never increased by convections, the values of the conserved variable remains in between maximum and minimum. The TVD schemes are defined by the choice of a flux limiter function, in the present methodology the CHARM limiter is used [4]. The CHARM limiter is a smooth function which helps to stabilize the numerical scheme. The Smagorinsky subgrid model was used as a closure for the subgrid stress in filtered momentum equations. The transport equations were integrated in time by an explicit low storage three stage RungeKutta method. The numerical accuracy of the present finite volume technique is second-order in space. For further details, please refer $[4-6,13]$.

\subsection{Theory and modeling of the filtered reaction rates in LES}

The Eddy Dissipation concept for the turbulence combustion proposed by Magnussen and Hjertager [14], Magnussen [11] and Gran and Magnussen [15] is a mixing controlled model. Performance of the EDC is highly dependent on the turbulence model. Turbulence contains eddies of different length and time scale. Larger eddies extract energy from the mean flow, these eddies are highly unstable and breakdown in smaller eddies. This breakdown process continues until eddies are sufficient small and they can not transfer energy further down. Eventually, this process creates structures that are small enough that molecular diffusion becomes important and viscous dissipation of kinetic energy finally takes place. The scale at which this happens is the Kolmogorov length scale. These dissipative eddies are termed as fine structures. In DNS these structures are explicitly calculated on the grid and does not require the combustion modeling. In RANS none of the eddies or structures are computed on the grid and the fine structures length and velocity scales are evaluated using the turbulent kinetic energy $k$ and its dissipation rate $\varepsilon$. The characteristic length $L^{\star}$ and velocity scale $u^{\star}$ of the fine structures based on the cascade theory of turbulence [16] are

$$
L^{\star}=\frac{2}{3}\left(\frac{3 C_{D 2}^{3}}{C_{D 1}^{2}}\right)^{1 / 4}\left(\frac{\nu^{3}}{\epsilon}\right)^{1 / 4}=1.43\left(\frac{\nu^{3}}{\epsilon}\right)^{1 / 4}
$$




$$
u^{\star}=\left(\frac{C_{D 2}}{3 C_{D 1}^{2}}\right)^{1 / 4}(\nu \epsilon)^{1 / 4}=1.75(\nu \epsilon)^{1 / 4}
$$

where $C_{D 1}=0.134$ and $C_{D 2}=0.5[11,14,16] . \nu$ is the kinematic viscosity and $\epsilon$ is eddy dissipation rate. The scales $L^{\star}$ and $u^{\star}$ are the same order of magnitude as the Kolmogorov length scales. The constant associated with Eq. (4) and (5) are developed assuming the full cascade process at each numerical cell. A connection between the fine structure and the larger eddies is achieved through the cascade. Large eddy simulation although resolves most of the eddies or structures but fine dissipative structures are not calculated due to computational constraint. The smallest length scale available in LES is a filter width. The size of the fine structure eddies is much smaller than the filter width and need to be computed as a function of filter width. In principle, these fine structure regions are embedded within highly strained eddies.

The transport equation Eq. (3) requires a closure for the source term. In EDC model, it is assumed that each numerical cell consists of a reactor, with inflow equal to surrounding mass fraction and outflow equal to burnt fine structures. The source term in the transport equation Eq. (3) with EDC model is

$$
\omega_{i}=\frac{\gamma_{\lambda}^{2} \chi}{\tau_{\star}}\left(Y_{i}^{0}-Y_{i}^{\star}\right),
$$

where $\gamma_{\lambda}$ is the fine structure region, $\gamma_{\lambda}^{2} / \tau_{\star}$ is the mass exchange between the surrounding and the fine structure region. Superscripts ${ }^{\star}$ and ${ }^{0}$ refer the fine structure and the surrounding state respectively. $\tau_{\star}$ is a residence or mixing time. $\chi[13]$ is a fraction of the fine structure where reaction takes place. The fine structures state $Y^{\star}$ are estimated using the reactor modeling. In the present study a perfectly stirred reactor (PSR) is used assuming that the reactor mass is constant at each time step and reactants are perfectly mixed inside it. The chemistry in the reactor can be modeled either with fast chemistry, equilibrium or chemical kinetics. In order to save computational time the fast chemistry approach is used.

The fine structure region is estimated as a function of fine structure velocity and the eddy characteristic velocity $u^{\prime}, \gamma_{\lambda}=\left(u_{\star} / u^{\prime}\right)$. Characteristics of the larger eddies such as the velocity $u^{\prime}$ and length scale $l^{\prime}$ are evaluated using the turbulence model such as $k-\epsilon$. In LES most of the turbulent kinetic energy is resolved on the grid scale and only a small amount of the subgrid kinetic energy is available explicitly. The fine structures region $\gamma_{\lambda}$ is evaluated using the subgrid eddy velocity $u_{s g s}$ and fine structure velocity $u_{\star}$.

$$
\gamma_{\lambda}=\left(\frac{u_{\star}}{u_{s g s}}\right)
$$

and using the Eq.(5), the fine structure region can be expressed as

$$
\gamma_{\lambda}=\left(\frac{3 C_{D 2}}{4 C_{D 1}^{2}}\right)^{1 / 4}\left(\frac{\nu \epsilon}{k_{s g s}^{2}}\right)^{1 / 4}
$$


where $k_{s g s}$ is the subgrid kinetic energy and is modeled $\nu_{s g s}=C_{k} \triangle k_{s g s}{ }^{1 / 2}$ and similarly the subgrid eddy dissipation rate is modeled $\epsilon_{s g s}=C_{\epsilon}\left(k_{s g s}\right)^{3 / 2} / \triangle$. The relationship between the subgrid viscosity, kinetic energy and dissipation is given by $\nu_{s g s}=C_{\nu s g s} k_{s g s}^{2} / \epsilon_{s g s}$. Most of the subgrid models in LES evaluate the eddy viscosity explicitly, and the subgrid kinetic energy and dissipation are calculated implicitly. Then the EDC model is formulated using the eddy viscosity $\nu_{s g s}$, where the dimensionless model coefficients are $C_{k}=0.05$ and $C_{\epsilon}=1.00$ [17]. The fine structure region is represented as

$$
\gamma_{\lambda}=\left(\frac{3 C_{\nu s g s} C_{D 2}}{4 C_{D 1}^{2}}\right)^{1 / 4}\left(\frac{\nu}{\nu_{s g s}}\right)^{1 / 4}
$$

Equation (8) is a function of the molecular viscosity, the subgrid viscosity and the model constants. In case of LES a model constant $C_{E D C}=$ $\left(3 C_{\nu s g s} C_{D 2} / 4 C_{D 1}^{2}\right)^{1 / 4}$ is introduced. Value of the model constant is $C_{E D C}=$ 1.01, where $C_{\nu s g s}=0.05$ [17]. Using this value of the $C_{E D C}$ overpredicts the temperature and the flame height is also under predicted. That is because the constant $C_{E D C}$ has been developed using the standard value of $C D_{2}$ and $C D_{1}$ which are based on the full turbulence energy cascading. The model constant $C_{E D C}$ has to be modified based on the partial cascading or no cascading in LES. In order to establish the constant $C_{E D C}$ parametric studies were carried out, and it was found that the model constant $C_{E D C}$ of 0.25 [13] gives better predictions. Although in the current formulation, EDC constant is assumed to be fixed, however in realistic problem the model constant has to be computed dynamically. In addition to that an upper limit of $\left(\gamma_{\lambda}<1\right)$ is used wherever the fine structure velocity is larger than the subgrid velocity.

\subsection{Density calculation for splitting the conserved variables}

In reacting flows, solution of the pressure correction Poisson equation with density time derivative term causes a severe time constraint per iteration and is the most destabilizing part of the calculation. This stability problem mostly dominates near to the nozzle due to sudden heat release. A major issue discussed by Kempf et al. [4], was the splitting of $\rho f$ with density $\rho$ in the flamelet approach. He proposed an Echt Konservativer Transport (EKT) scheme to alleviate this problem, which is based on the conservation of fuel $\rho f$ and oxidizer $\rho(1-f)$. The EKT scheme assumes two pseudo species $X_{A}=f^{+}=f$ and $X_{B}=f^{-}=(1-f)$ and the transport equations for the pseudo species were solved in predictor and corrector steps. In the EKT approach the density was formulated as [4]

$$
\bar{\rho}=\left(\bar{\rho} \widetilde{f}^{+}\right)^{n+1^{\star}}+\left(\bar{\rho} \widetilde{f}^{-}\right)^{n+1^{*}}
$$

A similar problem is also faced while splitting $\rho Y_{i}$ with density in the EDC approach [13]. Then a method similar to EKT is proposed for calculation of the 
density. In the present formulation, the species mass fractions were conserved instead of pseudo species:

$$
\bar{\rho}=\sum_{i=1}^{i=n}\left(\rho Y_{i}\right)^{n+1^{\star}} / \sum_{i=1}^{i=n}\left(Y_{i}\right)^{n+1^{\star}}
$$

where $\sum_{i=1}^{i=n}\left(Y_{i}\right)^{n+1^{\star}}=1$. The time derivative density term in the pressure correction equation is evaluated at each time step using the chemical density and density from Eq. (10)

$$
\frac{\partial \bar{\rho}}{\partial t}=\frac{\bar{\rho}_{c h e m}-\bar{\rho}}{\triangle t}
$$

\section{Results and discussion}

In the present study, a LES of the $\mathrm{H}_{2} / \mathrm{N}_{2}$ diffusion flames Flame $\mathrm{H} 3$ [18] was carried out. The studied configuration has a fuel nozzle with a diameter (D) of $8 \mathrm{~mm}$, which was surrounded by an air co-flow of $0.2 \mathrm{~m} / \mathrm{s}$. The fuel jet consist of a mixture of $50 \%$ of $\mathrm{H}_{2}$ and $50 \%$ of $\mathrm{N}_{2}$ by volume. The fuel bulk velocity was $34.8 \mathrm{~m} / \mathrm{s}$, which gave a Reynolds number of 10,000 based on the nozzle diameter. For the numerical simulation a cylindrical computational domain with $75 \mathrm{D}$ in axial direction and 25D in radial direction was employed. The numerical grids of $514 \times 32 \times 57$ were used axially, circumferentially and radially respectively. The grid was equidistant in the axial and circumferential directions. In the radial direction, 5 uniform cells were used in the nozzle, otherwise the grid was stretched linearly. The inflow boundary condition was Dirichlet condition. The mean velocity profiles superimposed with fluctuations were applied at the inflow. The fluctuations with specified length scales were generated using the turbulence inflow generator developed by Klein et al. [19]. On the downstream (outflow boundary) and the annular surface of the computational domain zero gradients for velocity components were posed. Periodicity was applied to all quantities in the circumferential direction.

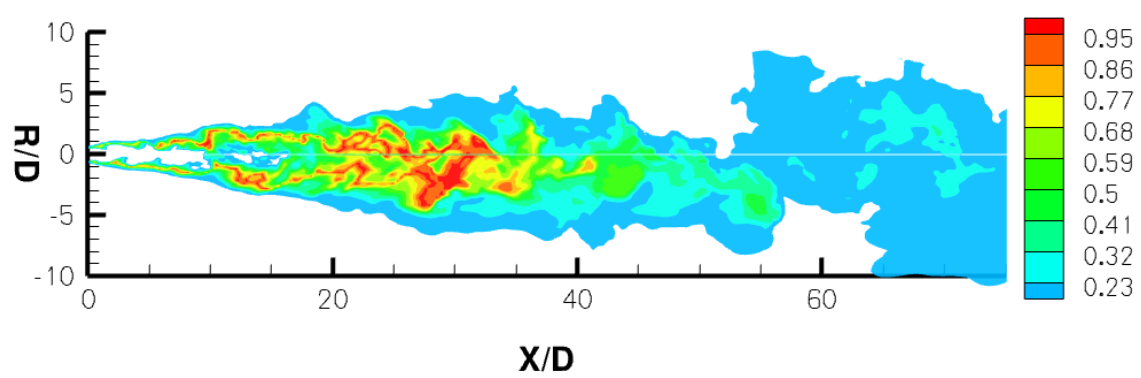

Figure 1: Instantaneous distribution of $\chi$. 

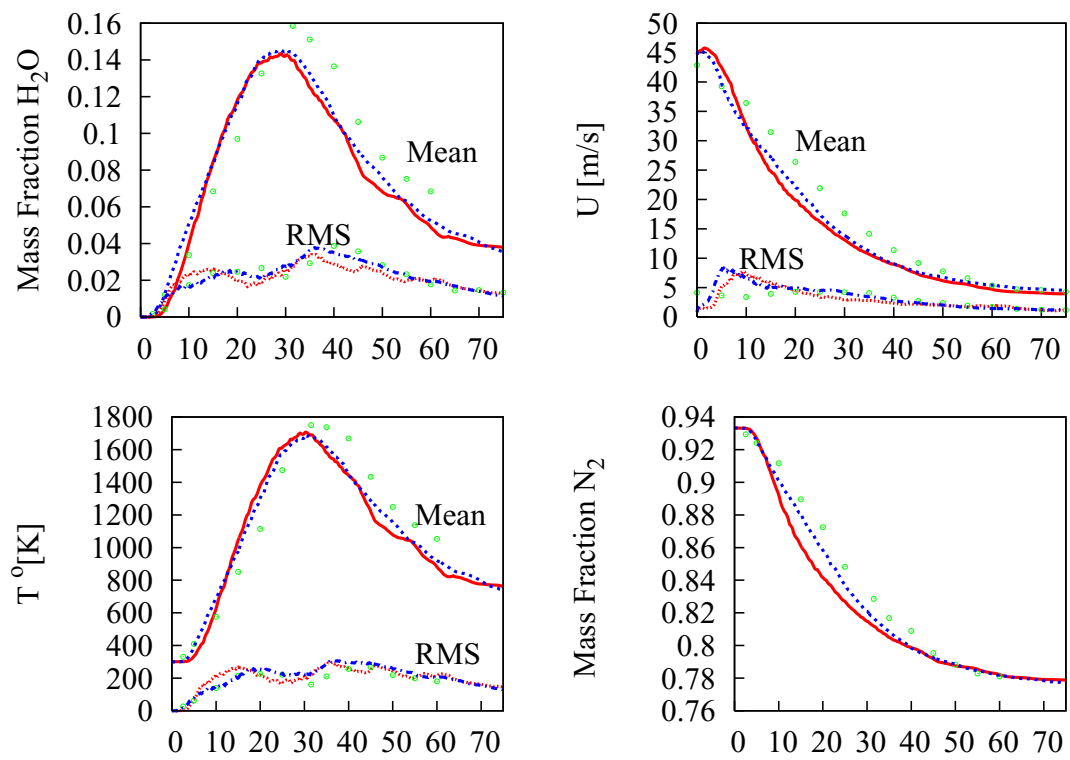

Figure 2: Mean and RMS centerline profiles of the mass fraction of $\mathrm{H}_{2} \mathrm{O}$, mass fraction of $\mathrm{N}_{2}$, temperature and velocity, where $\odot,--$ and _ represent experimental, Flamelet and EDC respectively.

The instantaneous plot for the $\chi$ is shown in fig. 1. It can be seen that the $\chi$ is comparatively higher, especially at the thin reaction zone between the jet and coflow. The main reaction zone which varies from $25 \mathrm{D}-40 \mathrm{D}$ is also well represented by $\chi$. The concept of $\chi$ is very similar to the reaction progress variable approach of Pierce and Moin [20]. Where a mixture fraction controls the mixedness of the fuel and oxidizer and the progress variable estimates a global extent of the reaction. Similarly, in the EDC model the fine structure mass fraction represents an amount of the mixed fuel and oxidizer, the extent of the reaction is governed by $\chi$. In the previous study $[10,21]$, where the earlier version of EDC model [11] without $\chi$ was used, the predictions were not satisfactory in comparison to the experiments. Simulations without $\chi$ tends to overpredict the temperature and major mass species fractions. LES, which is computationally demanding, reactor modeling of the fine structures with chemical kinetics makes still more expensive. The computational cost can be reduced by modeling the reactor with the fast chemistry assumption and with appropriate $\chi$. The time averaged and variance of the temperature, velocities and mass fractions of species is depicted in figs 2 and 3. The calculated mean and RMS mass fractions of $\mathrm{H}_{2} \mathrm{O}, \mathrm{N}_{2}$, and temperature profiles along the centerline is shown in fig. 2. The computed results are compared with the steady state flamelet model and experimental data base. The flame height with EDC model is predicted well. It is observed that the prediction of mean and variance is quite satisfactory with experiments. 


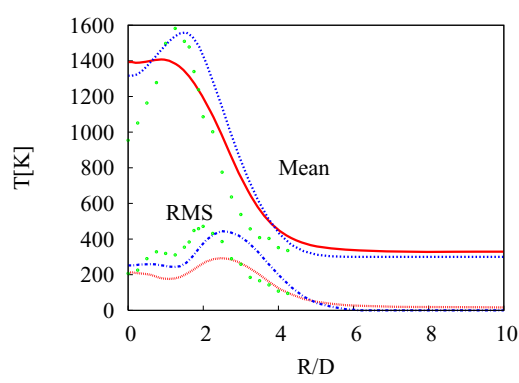

(a) $\frac{R}{D}=20$

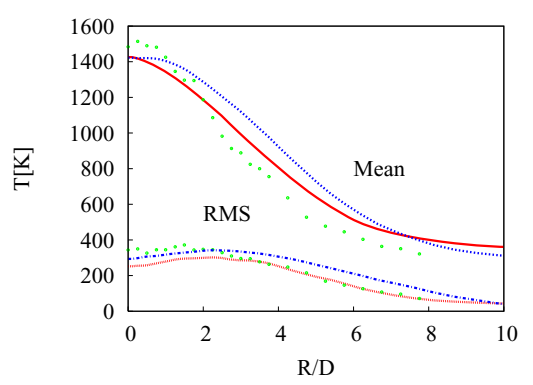

(c) $\frac{R}{D}=40$

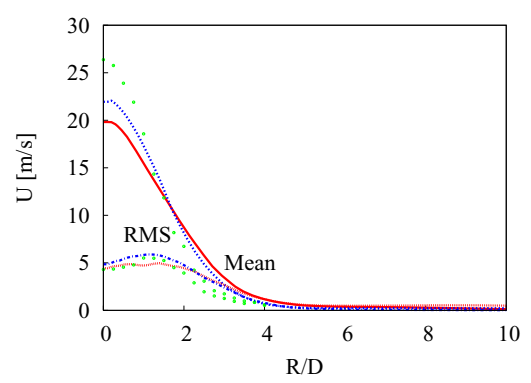

(b) $\frac{R}{D}=20$

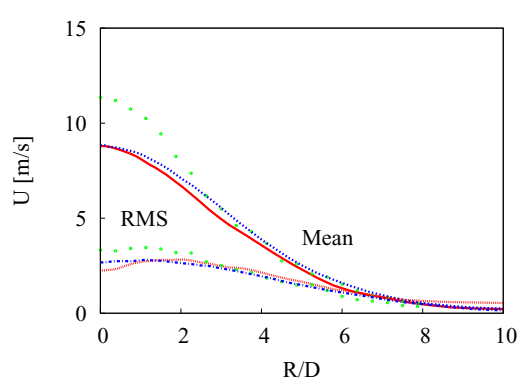

(d) $\frac{R}{D}=40$

Figure 3: Mean and RMS radial profiles of the temperature and velocity, where $\odot,--$ and $\_$represent experimental, Flamelet and EDC results respectively.

It can be seen from fig. 2 that the peak of the variance of velocity is high compared to the experiment. The instantaneous mixing caused a sudden heat release and thus expansion near to the nozzle, which caused the deceleration in the fluid. The sudden heat release also caused the dilatation within the thin mixing layer between the fuel and oxidizer, thereby pushing apart the fuel and oxidizer when they try to mix. This process could be transient in nature. The boundary condition at the inflow is Dirichlet fixed velocity, which does not allow for relaxation of the upstream flow. In experiments the boundary condition at the inlet is not the Dirichlet fixed velocity, the fluctuation due to the heat release will be adjusted automatically. The Dirichlet boundary condition is known to work well for non reactive flows. All these effects caused increased of fluctuations or turbulent kinetic energy near to the nozzle $[4,13]$, as observed in fig. 2. In addition to that, the central differencing scheme used for the convective terms produces high fluctuations, especially close to the nozzle. Simulating upstream nozzle using immersed boundary condition along with a higher order convective diffusive scheme might improve the solution.

The mean and RMS radial profiles of the calculated temperature and velocities at $R / D=20$ and $R / D=40$ are shown in fig. 3. The calculated values are 
compared with experiments and flamelet solutions and the comparison seems to be satisfactory. It is observed that the velocity was underpredicated at the core $R / D<1.5$ which affected the overall spread. A similar behavior is also observed with the flamelet approach. It can be concluded that this behavior is not because of the combustion model used but because of the inflow boundary condition and modeling of the nozzle.

\section{Conclusions}

A Turbulence Chemistry Interaction Model (TCIM) based on the Eddy Dissipation Concept (EDC) is proposed for large eddy simulation. The proposed model estimates the fine structure mass fraction using the subgrid viscosity and kinematic viscosity. Modification of EDC model constants $C_{E D C}=0.25$ was proposed to account for the partial turbulence energy cascading. The LES of the H2/N2 non-premixed unconfined turbulent jet flame is performed using LES-EDC and Flamelet model. The predicted means and variances of velocity and scalars are in satisfactory agreement to the experimental data and flamelet approach. A novel approach for estimating the density is proposed, where density is formulated from species mass fraction, which is numerically stable and computationally less expensive. Proper modeling of the inflow boundary conditions and nozzle is essential for obtaining the accurate predictions.

\section{Acknowledgements}

This work has been carried out as a part of Institution-based Strategic Project (ISP) funded by The Research Council of Norway. This support is gratefully acknowledged. We are indebted to Professor Emeritus Bjørn Magnussen for discussions and suggestions on the EDC model. We are also thankful to TU Darmstadt for providing the source code of FLOWSI code.

\section{References}

[1] Landenfeld, T., A turbulence-chemistry interaction model based on a multivariate presumed beta-pdf method for turbulent flames. Applied Scientific Research, 68, pp. 111-135(25), March 2002.

[2] Branley, N. \& Jones, W.P., Large eddy simulation of a turbulent nonpremixed flame. Combustion and Flame, 127(1-2), pp. 1914 - 1934, 2001.

[3] Forkel, H. \& Janicka, J., Large-eddy simulation of a turbulent hydrogen diffusion flame. Flow, Turbulence and Combustion, 65(2), pp. 163-175, 2000.

[4] Kempf, A., Large Eddy Simulation of Non-Premixed Turbulent Flames. Ph.D. thesis, Darmstadt University of Technology, Darmstadt, Germany, 2003. 
[5] Kempf, A., Lindstedt, R.P. \& Janicka, J., Large-eddy simulation of a bluff-body stabilized nonpremixed flame. Combustion and Flame, 144(1-2), pp. 170-189, 2006.

[6] Kempf, A., Malalasekera, W., Ranga-Dinesh, K. \& Stein, O., Large eddy simulations of swirling non-premixed flames with flamelet models: A comparison of numerical methods. Flow, Turbulence and Combustion, 81(4), pp. 523-561, 2008.

[7] Pitsch, H. \& Steiner, H., Large-Eddy Simulation of a turbulent piloted methane/air diffusion flame. APS Meeting Abstracts, 1999.

[8] McMurtry, P.A., Menon, S. \& Kerstein, A.R., Linear eddy modeling of turbulent combustion. Energy \& Fuels, 7(6), pp. 817-826, 1993.

[9] Kerstein, A., Linear-eddy modelling of turbulent transport. Part 7. Finiterate chemistry and multi-stream mixing. Journal of Fluid Mechanics, 240, pp. 289-313, 1992.

[10] Hu, L.Y., Zhou, L.X. \& Zhang, J., Large-eddy simulation of a swirling diffusion flame using a som sgs combustion model. Numerical Heat Transfer, Part B: Fundamentals: An International Journal of Computation and Methodology, 50(1), pp. 41-58, 2006.

[11] Magnussen, B.F. \& Hjertager, B., On mathematical modeling of turbulent combustion with special emphasis on soot formation and combustion. 16th Symp. (Int.) on Combustion (1976). Comb. Inst., Pittsburg, Pennsylvania, pp. 719-729.

[12] Yaga, M., Endo, H., Yamamoto, T., Aoki, H. \& Miura, T., Modeling of eddy characteristic time in LES for calculating turbulent diffusion flame. International Journal of Heat and Mass Transfer, 45(11), pp. 2343-2349, 2002.

[13] Panjwani, B., Ertesvåg, I.S., Gruber, A. \& Rian, K.E., Subgrid combustion modeling for large eddy simulation (les) of turbulent combustion using eddy dissipation concept. Fifth European Conference on Computational Fluid Dynamics, ECCOMAS CFD 2010,Lisbon, Portugal, 2010.

[14] Magnussen, B.F., On the structure of turbulence and a generalized eddy dissipation concept for chemical reaction in turbulent flow. 19 AIAA Aerospace Science Meeting, 1981.

[15] Gran, I.R. \& Magnussen, B.F., A numerical study of a bluff-body stabilized diffusion flame. part 2. influence of combustion modeling and finite-rate chemistry. CombSciTech, 119, pp. 191-217, 1996.

[16] Ertesvåg, I.S. \& Magnussen, B.F., The eddy dissipation turbulence energy cascade model. Combustion Science and Technology, 159(1), pp. 213-235, 2000.

[17] Fureby, C., Tabor, G., Weller, H.G. \& Gosman, A.D., A comparative study of subgrid scale models in homogeneous isotropic turbulence. Physics of Fluids, 9(5), pp. 1416-1429, 1997.

[18] Http://www.tu-darmstadt.de/fb/mb/. 
[19] Klein, M., Sadiki, A. \& Janicka, J., A digital filter based generation of inflow data for spatially developing direct numerical or large eddy simulations. J. Comput. Phys., 186(2), pp. 652-665, 2003.

[20] Pierce, C.D. \& Moin, P., Progress-variable approach for large-eddy simulation of non-premixed turbulent combustion. Journal of Fluid Mechanics, 504(-1), pp. 73-97, 2004.

[21] Zhou, L.X., Hu, L.Y. \& Wang, F., Large-Eddy Simulation of Turbulent Combustion Using SOM and EBU SGS Combustion Models, pp. 99-102, 2009 . 